\title{
Temperature Dependence of the Dissociation Width of Dislocations in a Commercial 304L Stainless Steel
}

\author{
M. FUJITA, Y. KANEKO, A. NOHARA, ${ }^{1)}$ H. SAKA, ${ }^{1)}$ R. ZAUTER ${ }^{2)}$ and H. MUGHRABI ${ }^{3)}$
}

Department of Materials Science and Engineering, Nagoya University, Furo-cho, Chikusa-ku, Nagoya, Aichi-ken, 464-01 Japan. $\quad 1$ ) Department of Quantum Engineering, Nagoya University, Furo-cho, Chikusa-ku, Nagoya, Aichi-ken, 464-01 Japan. $\quad 2$ 2) Formerly Institut für Werkstoffwissenschaften, Universität Erlangen-Nürnberg. Now at Babcok Rohrleitungsbau $\mathrm{GmbH}, 46049$ Oberhausen, Federal Republic of Germany. 3) Institut für Werkstoffwissenschaften, Universität Erlangen-Nürnberg, 91058 Erlangen, Federal Republic of Germany.

(Receivèd on March 16, 1994; accepted in final form on May 25, 1994)

\begin{abstract}
The dissociation width of dislocations in a commercial AISI $304 \mathrm{~L}$ stainless steel was measured as a function of temperature. Two types of experiments were carried out. In one, specimens were deformed at room temperature, followed by annealing at various temperatures, and in the other, specimens were deformed directly at various temperatures. In both experiments the width of near-edge dislocations remained unchanged upto $300-400^{\circ} \mathrm{C}$, then decreasing gradually. On the other hand, the width of near-screw dislocations showed the maximum at around $400^{\circ} \mathrm{C}$, when specimens were deformed directly at high temperatures: When specimens were deformed at room temperature and then annealed at high temperatures, the maximum was less evident. The Suzuki segregation was invoked to explain behaviour of the dissociated dislocations in a commercial AISI 304L stainless steel.

It is proposed that some typical features of dynamic strain ageing observed at around $400^{\circ} \mathrm{C}$ such as enhanced work hardening and planar slip could be caused in part and in conjunction with other more effective mechanisms than those caused by the slightly enhanced dissociation width of near-screw dislocations in the same temperature range.
\end{abstract}

KEY WORDS: dissociated dislocations; 304L stainless steel; Suzuki effect.

\section{Introduction}

Austenitic stainless steels are of considerable practical significance, in particular for applications at temperatures upto about $600^{\circ} \mathrm{C}$. In this context, it is interesting to note that austenitic stainless steels exhibit pronounced dynamic strain ageing effects at temperatures between 200 and $600^{\circ} \mathrm{C}^{1-3)}$ In recent years, several studies have focussed on dynamic strain ageing effects during fatigue at elevated temperatures. ${ }^{4-6)}$ An important observation in these studies relates to the effect of dynamic strain ageing on the slip mode of deformation. Thus, it was found that, with increasing temperature and enhancement of dynamic strain ageing, the flow stress increases and goes through a maximum at around $400^{\circ} \mathrm{C}$. At the same time, the dislocation slip mode changes from an initially so-called wavy slip mode to a so-called planar slip mode in the temperature range of maximum dynamic strain ageing and back to a wavy slip mode at higher temperatures. ${ }^{5,6)}$

Several processes must be considered as possible factors responsible for the described changes of slip model. Among these, the ease of cross slip related to the stacking fault energy (SFE) ${ }^{7)}$ or the dissociation width of dislocations, the cutting of small coherent car- bides $^{5)}$ and facilitation of planar slip by short range ordering processes ${ }^{8)}$ are considered to be the most important factors.

In the present study, the importance of the first of these three mechanisms will be considered. For this purpose, the temperature dependence of the dissociation width of dislocations (or equivalently the SFE) of AISI 304L stainless steel will be investigated.

Briefly, our present knowledge on the SFE of austenitic stainless steels is as follows. Rhodes and Thompson ${ }^{9)}$ have studied the concentration dependence of the SFE in $\mathrm{Fe}-\mathrm{Cr}-\mathrm{Ni}$ austenitic stainless steel $(0.04 \mathrm{wt} \% \mathrm{C})$. They concluded that the concentration dependence of the SFE in austenitic stainless steel is rather complicated. Bampton et al. ${ }^{10)}$ using weak-beam electron microscopy, studied the effects of annealing on the room temperature value of the SFE in some low carbon $\mathrm{Fe}-\mathrm{Cr}-$ $\mathrm{Ni}$ austenitic stainless steel $(<0.02 \mathrm{wt} \% \mathrm{C})$. They did not detect any difference in the apparent SFE between fresh dislocations (i.e., those introduced by room-temperature deformation) and annealed ones over the whole range of the dislocation characters. Latanision and Ruff $^{11)}$ and Lecroisey and Pineau ${ }^{12)}$ have carried out in-situ heating experiments in an electron microscope on low carbon $\mathrm{Fe}-\mathrm{Cr}-\mathrm{Ni}$ austenitic stainless steel $(<0.01$ 
wt $\% \mathrm{C}$ ), and have shown that the SFE increases with temperature in a reversible manner. Abrassart ${ }^{13}$ ) has carried out similar in-situ heating experiment on high carbon $(0.131-0.18 \mathrm{wt} \% \mathrm{C}) \mathrm{Fe}-\mathrm{Cr}-\mathrm{Ni}$ austenitic stainless steels and has shown that the SFE apparently increased with increasing temperature during the first run of heating and that it did not recover to the original value after cooling to room temperature.

So far, the temperature dependence of the SFE in a commercial $\mathrm{Fe}-\mathrm{Cr}-\mathrm{Ni}$ austenitic stainless steel with medium carbon content has not been investigated. The present study reports the result about the temperature dependence of the SFE in AISI 304L stainless steel obtained by comprehensive electron microscopy of dissociated dislocations.

\section{Starting Materials}

The chemical composition of the austenitic steel AISI 304L used in this study is given in Table 1. Prior to machining of the specimens from bars with a diameter of $25 \mathrm{~mm}$, a two-step heat treatment was applied: In the first step, the alloy was solution heat-treated by annealing at $1100^{\circ} \mathrm{C}$ for $3 \mathrm{hr}$. After quenching in water it was annealed subsequently at $600^{\circ} \mathrm{C}$ for $1000 \mathrm{hr}$.

\section{Experimental Procedures}

Two types of experiments were carried out. 1) Deformation at different temperatures + room temperature observation, 2) Room temperature deformation + bulk annealing + room temperature observation.

\subsection{Deformation at Different Temperature + Room Temperature Observation}

Specimens for tensile tests were machined with a gauge length 10 and $8 \mathrm{~mm}$ diameter. They were deformed in tension to fracture at a strain rate of $6.7 \times 10^{-4} \mathrm{~s}^{-1}$ at $250,400,525$ and $650^{\circ} \mathrm{C}$ on an Instron tensile testing machine. Specimens deformed to fracture were sectioned into discs using a multi-wire abraser saw in such a way that the normals of the discs are inclined to the tensile axis by $45^{\circ}$. The discs were then electropolished to electron transparency using a Tenupol automatic thinner.

\subsection{Room Temperature Deformation + Bulk Annealing + Room Temperature Observation}

At room temperature martensitic transformation was induced by a severe deformation, so that $3 \mathrm{~mm}$ discs were bent lightly by hands to introduce dislocations. These discs were annealed at $250,300,400$ and $525^{\circ} \mathrm{C}$ for $30 \mathrm{~min}$, followed by water quenching. Foil specimens were prepared by electropolishing in a Tenupol automatic thinner.

Table 1. Chemical composition of AISI 304L under investigation in $w t \%$.

\begin{tabular}{cccccccc}
\hline $\mathrm{C}$ & $\mathrm{Cr}$ & $\mathrm{Ni}$ & $\mathrm{Mn}$ & $\mathrm{Si}$ & $\mathrm{S}$ & $\mathrm{P}$ & $\mathrm{Fe}$ \\
\hline 0.024 & 18.1 & 8.95 & 1.44 & 0.62 & 0.007 & 0.038 & bal. \\
\hline
\end{tabular}

\subsection{Electron Microscopy}

Foil specimens were examined in the JEOL $200 \mathrm{CX}$ electron microscope at an accelerating voltage of $200 \mathrm{kV}$. Since the specimens were polycrystalline, only those grains whose normals happened to be close to the primary (111) plane were studied. Dislocations were imaged in the weak-beam mode using 220 reflections. Only long, isolated, nearly straight dislocations lying in the plane of foil were studied.

\section{Results}

\subsection{Deformation at Different Temperatures + Room Temperature Observation}

Figure 1 shows the temperature dependence of the normalized stress increment by work hardening of the tensile-deformed specimens investigated in this study. The normalized stress increment at a temperature $T$ is defined as the difference between the stress after a given strain and the $0.2 \%$ offset stress, multiplied by the quotient of the Young's moduli at room temperature and at the temperature $T$, respctively. The curves in Fig. 1, referring to strains of 10 and $15 \%$ respectively, exhibit a maximum at about $400^{\circ} \mathrm{C}$, indicating that dynamic strain ageing has its maximum effect at this temperature.

Figure 2 shows high magnification weak-beam micrographs of dissociated dislocations introduced by deformation at $400^{\circ} \mathrm{C}$. In Fig. 2(a) the dislocations (denoted by $1,2,2^{\prime}$ and 3 ) were imaged in $g=220$, while in Fig. 2(c) they were imaged in $g=202$. In both cases only either of the two Shockley partial dislocations appeared strongly. In contrast, in Fig. 2(b) the dislocations were imaged in $g=022$ and both of the Shockley partial dislocations appeared strongly. Thus, the Burgers vector of the dissociated dislocations could be determined without ambiguity.

The actual dissociation width $\Delta$ was calculated from the experimental width $\Delta_{\mathrm{obs}}$ according to the equation proposed by Cockayne et al..${ }^{14)}$ and plotted against the orientation of a dislocation line $\phi$, where $\phi$ is the angle between the dislocation line and the total Burgers vec-

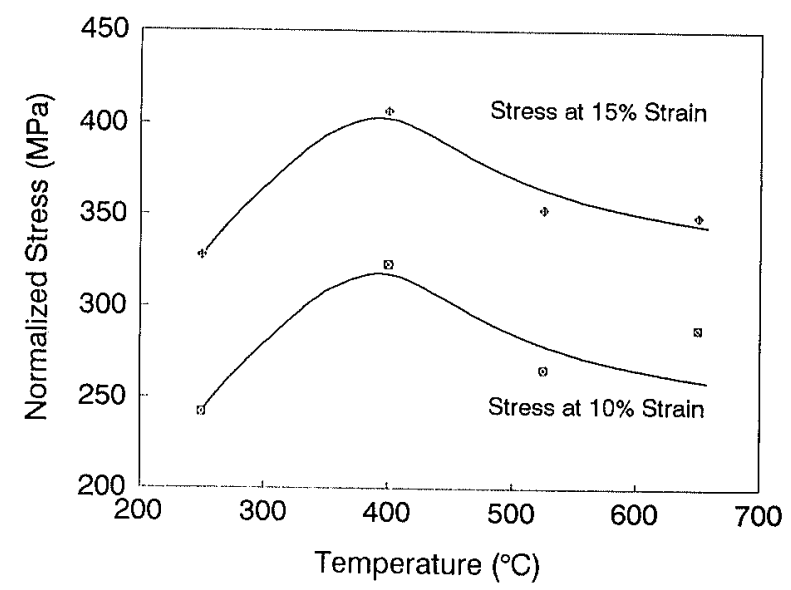

Fig. 1. Temperature dependence of the normalized stress increment by work hardening of tensile-deforemed AISI $304 \mathrm{~L}$ stainless steel at strains of $10 \%$ and $15 \%$, respectively (strain rate: $6.7 \times 10^{-4} \mathrm{~s}^{-1}$ ). 


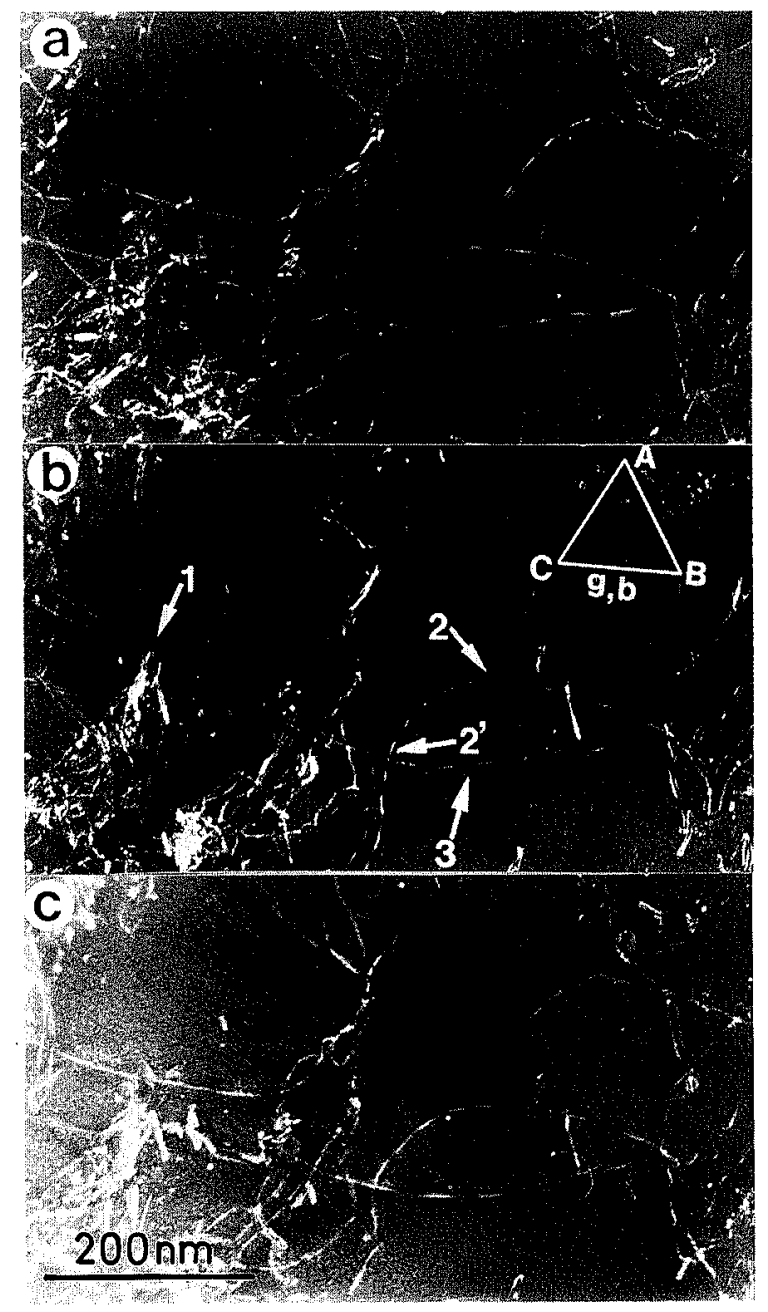

Fig. 2. High-magnification weak-beam micrograph showing dislocations introduced by deformation at $400^{\circ} \mathrm{C}$. In (a) dislocations $1,2,2^{\prime}$ and 3 are imaged in $\mathbf{g}=220$, in (b) they are imaged in $\mathbf{g}=202$. and in (c) they are imaged in $\mathbf{g}=022$. Only in (b) both of the partials of the dislocations appear strongly.

tors. An example is reproduced in Fig. 3, which refers to observations on a specimen deformed at room temperature. Theoretical curve calculated using anisotropic elasticity of dislocations ${ }^{15}$ ) with elastic constants taken from Salmutter and Stangler ${ }^{16)}$ is also plotted. Experimental observations fit very well with the theoretical curve. Figure 4 shows some examples of the widths of dissociated dislocations introduced by deformation at various temperatures $(250,400,525$ and $650^{\circ} \mathrm{C}$ ). At room temperature experimental data fit well with theoretical curves over the whole range of $\phi$, while at temperatures higher than $250^{\circ} \mathrm{C}$, data do not appear to fit well with the theoretical curves; those for $\phi<30^{\circ}$ (that is, near-screw dislocations) lie much higher than the theoretical lines extrapolated from the higher angles (that is near-edge dislocations).

The dissociation widths of those dislocations that were introduced directly by deformation at high temperatures are plotted in Fig. 5 for near-edge (Fig. 5(a)) and for near-screw dislocations (Fig. 5(b)) as a function of deformation temperature.

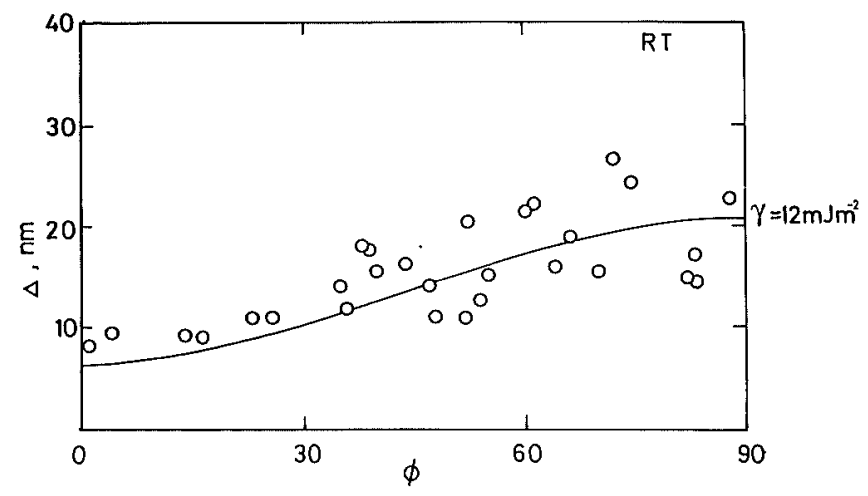

Fig. 3. The dissociation width plotted against $\phi$, the character of the total dislocations in a specimen deformed lightly at room temperature. Solid line shows the theoretical curve calculated by anisotropic elasticity.

\subsection{Room Temperature Deformation + Bulk Annealing + Room Temperature Observation}

Figure 6 shows $\Delta-\phi$ curves for those dislocations which were introduced by room-temperature deformation, followed by annealing at higher temperatures. In this case, experimental data fit fairly well with theoretical curves over the whole range of $\phi$. The dissociation widths of those dislocations that were introduced by deformation at room temperature, followed by annealing at high temperatures are plotted in Fig. 7 as a function of the annealing temperature.

\subsection{Comparison of Behaviour of Near-edge Dislocations and Near-screw Dislocations}

Comparison of Fig. 5 with Fig. 7 reveals the following features. The dissociation width of the near-edge dislocations remained unchanged upto $300-400^{\circ} \mathrm{C}$, then decreasing gradually with increasing temperature, regardless of whether the dislocations were introduced by room-temperature deformation or high-temperature deformation.

On the other hand, the behaviour of near-screw dislocations depended on whether they were introduced by room-temperature deformation or high-temperature deformation. The dissociation width of those near-screw dislocations that had been introduced by deformation at room temperature remained unchanged upto $400^{\circ} \mathrm{C}$, then decreasing with temperature. However, the dissociation width of near-screw dislocations that had been introduced by deformation at elevated temperatures shows a maximum at around $400^{\circ} \mathrm{C}$.

\section{Discussion}

\subsection{Factors Affecting the Dissociation Width}

The dissociation behaviour of a dislocation in f.c.c. alloys during a thermal cycling has been discussed in detail taking into account those factors which may give rise to the apparent temperature dependence of the SFE. ${ }^{13,17-21)}$ Among these, the most important factors include the following.

1) A True Dependence of the SFE on Temperature

We assume that the intrinsic SFE in an austenitic stainless steel increases with temperature. ${ }^{11,12)}$ 

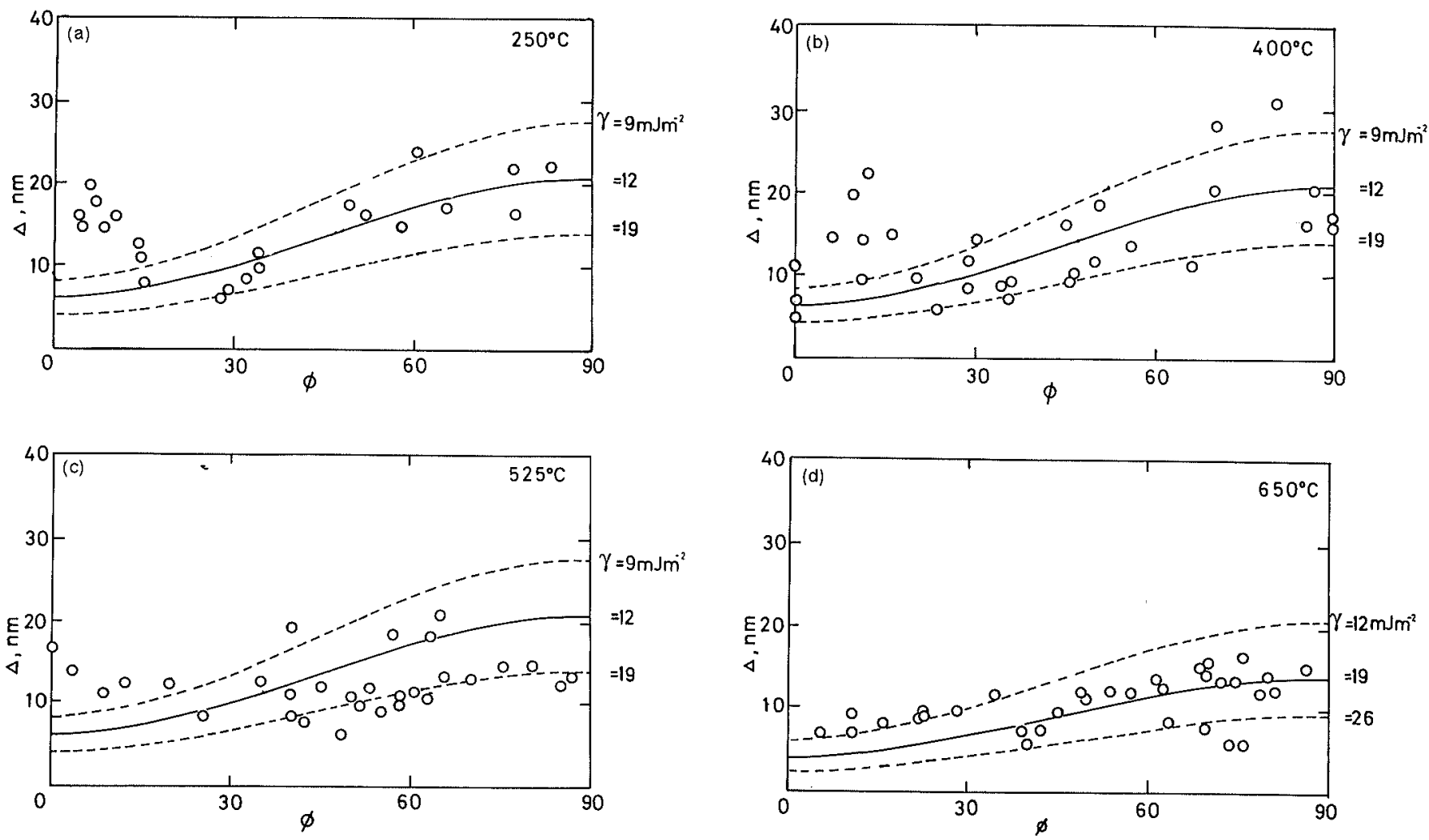

Fig. 4. Some examples of the widths of dissociated dislocations in specimens which were deformed at various temperatures. The number appearing in the top right-hand corner of each diagram refers to the temperature.
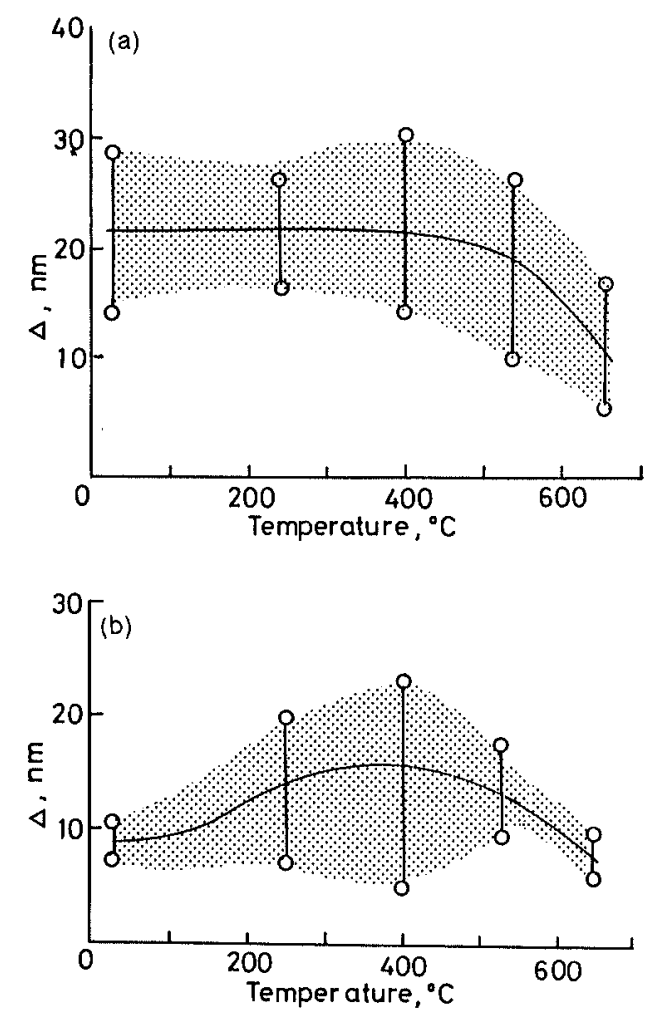

Fig. 5. The dissociation widths of dislocations introduced by deformation at high temperatures. (a) near-edge dislocations. (b) near-screw dislocations.

\section{2) Solute Impedance Effect}

Interaction between individual Shockley partials and solute atoms impedes the displacements of dislocations. We define a characteristic temperature, $T_{c}$, below which the individual Shockley partial dislocations are locked at the original positions due to the solute impedance effect.

\section{3) Suzuki Effect}

Chemical interaction between solute atoms and the stacking fault may result in the segregation of the solute atoms on the stacking fault.

We define two characteristic temperatures, $T_{d}$ and $T_{s}$. Here, $T_{d}$ is the temperature above which the diffusion of the solute atoms becomes substantial, and $T_{s}$ is the temperature above which the amount of segregation of the solute atoms is negligibly small.

In the present case at least three kinds of solute atoms must be taken into account, i.e., $\mathrm{Cr}, \mathrm{Ni}$ and $\mathrm{C}$. For $\mathrm{Cr}$ and $\mathrm{Ni}, T_{\mathrm{c}}$ is believed to be very low. This can be rationalized by the fact that the apparent SFE in low-carbon stainless steel varies in a reversible manner with temperature even below room temperature. ${ }^{11,12)}$ On the other hand, in a medium carbon stainless steel under consideration the dissociation width of near-edge dislocations remained unchanged after annealing at $300^{\circ} \mathrm{C}$, above which it decreased gradually. Thus, it can be inferred that $T_{c}$ for carbon for near-edge dislocations is around $300^{\circ} \mathrm{C}$. It is noted that $T_{c}$ for carbon for near-screw dislocations should be much lower, because the interaction energy between carbon and screw dislocations is much smaller than that between carbon and edge dislocations. In other words, in a medium carbon stainless steel as in the case of the present study, edge dislocations are likely subject to the solute impedance effects upto around $300^{\circ} \mathrm{C}$, while screw dislocations are not. 

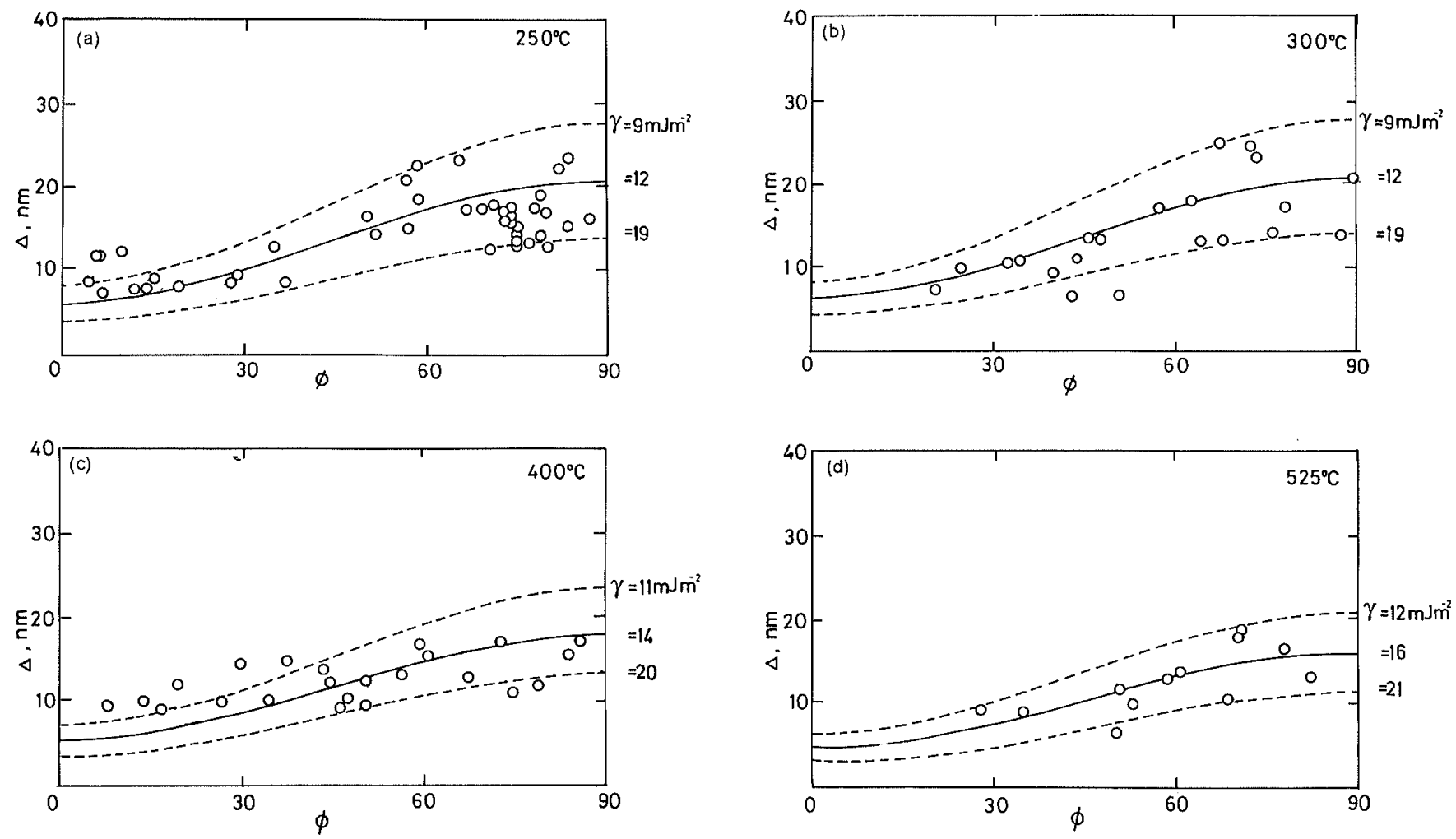

Fig. 6. Some examples of the widths of dissociated dislocations in specimens which were deformed at room temperature, followed by annealing at high temperatures. The number appearing in the top right-hand corner of each diagram refers to the temperature.
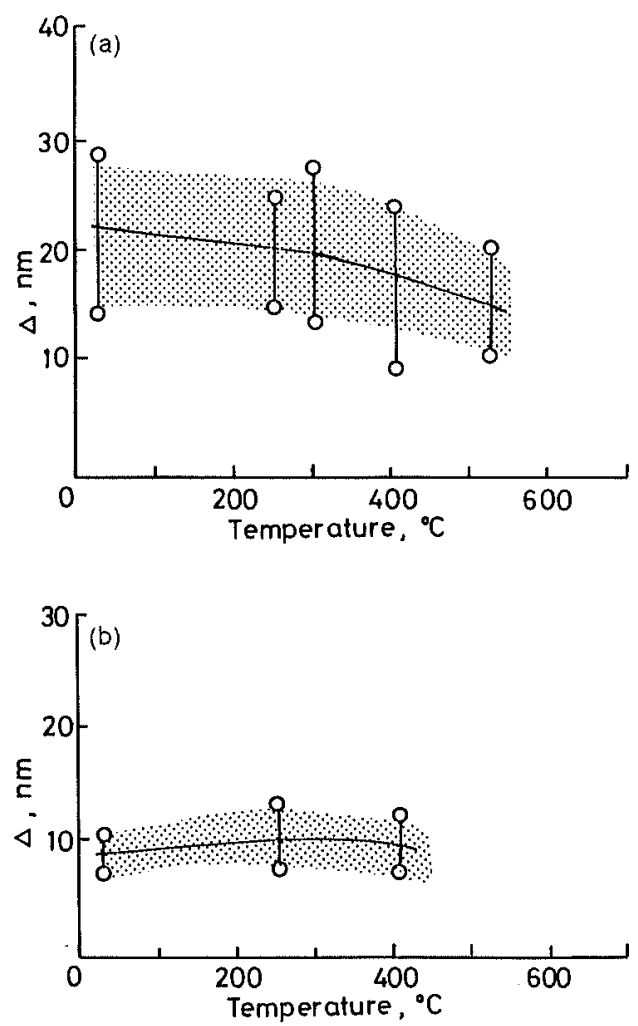

Fig. 7. The dissociation width of dislocations which were introduced by deformation at room temperature, followed by annealing at high temperature. (a) nearedge dislocations, (b) near-screw dislocations.
Next, we consider $T_{d} . T_{d}$ is the temperature above which the atomic diffusivity becomes significant. Since the diffusion is due to vacancies, when a large amount of point defects in excess of the thermal equilibrium concentration are introduced into the crystals by a severe deformation, the diffusion is expected to be enhanced. On the other hand, when crystals are annealed at high temperatures (after light deformation at room temperature), only thermal vacancies are available for the diffusion. In other words, deformation-enhanced diffusion must be taken into account when dislocations are introduced by severe high-temperature deformation.

\subsection{Dissociation Behaviour of Screw Dislocations}

First we consider the dissociation behaviour of screw dislocations, for which $T_{c}$ is well below room temperature. When screw dislocations are introduced by room-temperature deformation and the specimen is heated subsequently, the dissociation width will follow the curve for the unsegregated dislocations (indicated by dash-dotted line in Fig. 8(a)) until $T_{d}$ is reached, above which the width transits from the curve for the unsegregated dislocations to the curve for the segregated dislocations (broken line).* If $T_{d}$ is close to or higher than $T_{s}$, the transition does not take place; in such -a case, on cooling, the dissociation width follows the solid line in a reversible manner (indicated by (a)). In other words, the room-temperature value of the dissociation width after the complete thermal cycle is the same as the

* We do not know which solute atom segregates toward or desegregates away from the stacking fault to establish the equilibrium. We just assume that the dissociation width of segregated dislocations is larger than that of unsegregated dislocations. 

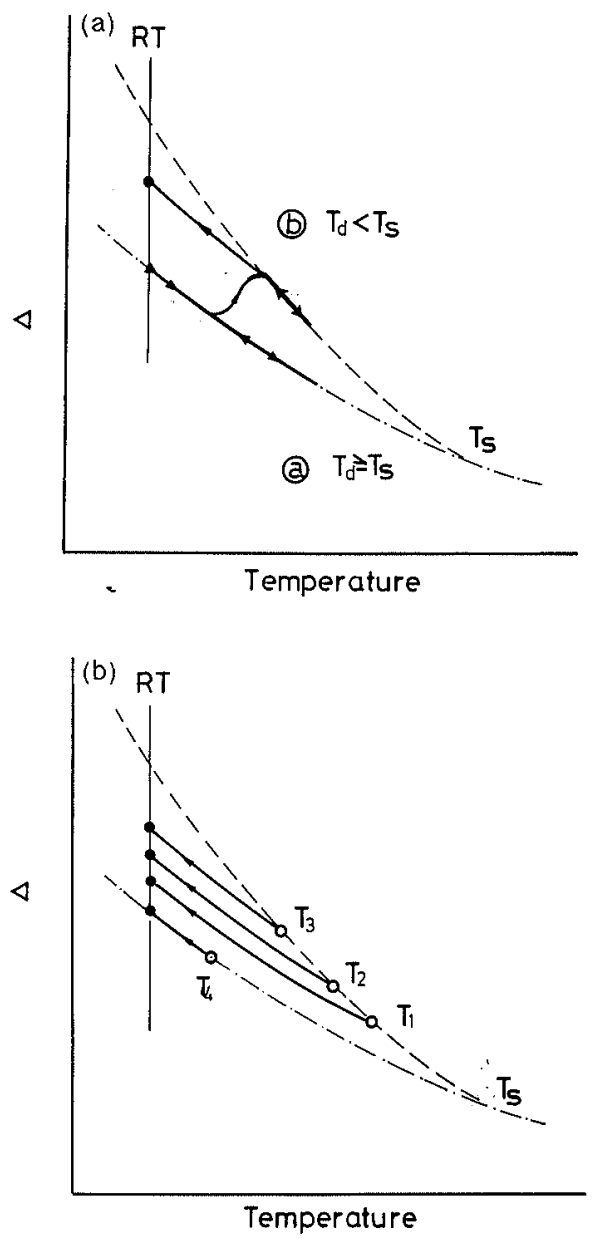

Fig. 8. Schematic illustration of the suggested mechanism by which the dissociation width of near-screw dislocations varies, (a) when a specimen is deformed at room temperature and then subjected to a thermal cycle and (b) when a specimen is deformed at high temperature and then cooled to room temperature. The broken line indicates the temperature dependence of the width of segregated dislocations, while the dash-dotted line indicates the temperature dependence of the width of unsegregated dislocations, due to the intrinsic temperature dependence of the stacking-fault energy. The solid lines indicate the actual variation of the width. $\Delta$ indicates the dissociation width obtained from dislocations introduced at room temperature.

as-deformed value. This is exactly what is observed. If $T_{d}$ were lower than $T_{s}$, then the room-temperature value after the complete thermal cycle should be higher than the original as-deformed value of the dissociation width (case (b)). But, this is not the case (cf. Fig. 7(b)). Thus, it can be said that $T_{d}$ is close to or higher than $T_{s}$ (case (a)). When screw dislocations are introduced by a heavy deformation at high temperatures, diffusion may take place during deformation even below $T_{d}$ because of the deformation-enhanced diffusion. Thus, it is reasonable to assume that the segregation of solute atoms towards dissociated dislocations takes place, i.e., the dissociation width of screw dislocations just after the deformation at high temperatures is in an equilibrium state. In other words, the dissociation width lies on the curve for the segregated dislocations $\left(T_{1}, T_{2}\right.$ and $T_{3}$ on the broken line in Fig. 8(b)). During cooling, however, the concentration of point defects available for diffusion will
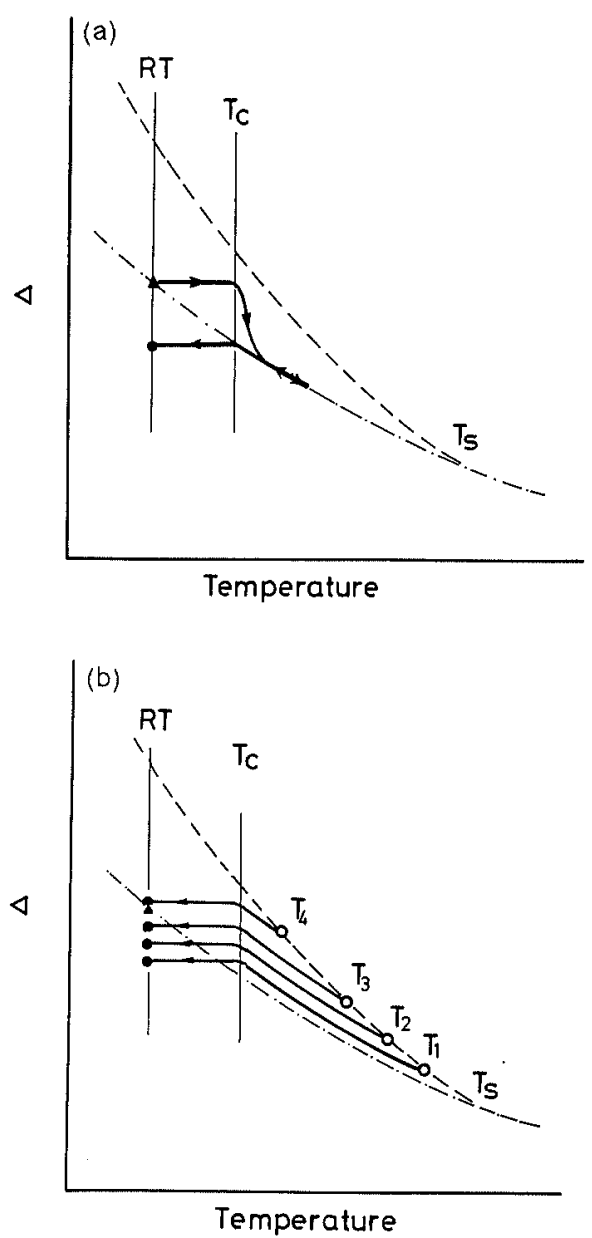

Fig. 9. Schematic illustration of the suggested mechanism by which the dissociation width of near-edge dislocations varies, (a) when a specimen is deformed at room temperature and then subjected to a thermal cycle and (b) when a specimen is deformed at high temperature and then cooled to room temperature. The broken line indicates the temperature dependence of the width of segregated dislocations, while the dash-dotted line indicates the temperature dependence of the width of unsegregated dislocations, due to the intrinsic temperature dependence of the stacking-fault energy. The solid lines indicate the actual variation of the width. $\triangle$ indicates the dissociation width obtained from dislocations introduced at room temperature.

quickly return to a thermal equilibrium one; no further segregation of solute atoms during cooling is expected. Thus, on cooling, the dissociation width follows the curves for partially segregated dislocations (indicated by solid lines in Fig. 8(b)), which are approximately parallel to the curve of the unsegregated dislocations (dash-dotted line). Therefore, the room-temperature value of the dissociation width of screw dislocations corresponds to the value of partially segregated dislocations.

When the deformation temperature is too low, the deformation-enhanced diffusion will not take place, so that the dissociation width lies on the curve for unsegregated dislocations $\left(T_{4}\right.$ on the dash-dotted line). Thus, the room-temperature value of the dissociation width after deformation above room temperature is to show the maximum between room temperature and $T_{s}$. Again, this is exactly what is observed (Fig. 5(b)). 


\subsection{Dissociation Behaviour of Edge Dislocations}

Next we consider the dissociation behaviour of edge dislocations, for which $T_{c}$ is above room temperature.

When edge dislocations introduced by the roomtemperature deformation are heated, the width remained unchanged because of the interaction between carbon and Shockley partials (solute impedance effect) until $T_{c}$ is reached, after which it tries to transit to the curve for the unsegregated dislocations (dash-dotted line in Fig. 9(a)). On cooling, the width decreases in a reversible manner above $T_{c}$, below which it is fixed to the value at $T_{c}$ again because of the solute impedance effect by carbon. Thus, the room-temperature value of the dissociation width of edge dislocations after the complete thermal cycle is similar to or smaller than the original as-deformed value, depending on whether the specimen is heated below $T_{c}$ or above $T_{c}$. This is exactly what is observed (Fig. 5(b)).

When edge dislocations are introduced by a heavy deformation at high temperatures, the segregation of solute atoms to the dissociated dislocations takes place because of the deformation-enhanced diffusion of solute atoms. Thus, the dissociation width lies on the curve for the segregated dislocations (broken line in Fig. 9(b)). On cooling, the dissociation width follows the curves for the partially segregated dislocations (indicated by solid line in Fig. 9(b)), which are approximately parallel to the curve of the unsegregated dislocations above $T_{c}$, just like in the case of screw dislocations introduced by high-temperature deformation. However, below $T_{c}$ the dissociation width is fixed to the value at $T_{c}$ because of the solute impedance effect by carbon. Thus, the room-temperature value of the dissociation width corresponds to the value of partially segregated dislocations at $T_{c}$, so that the width decreases with temperature above $T_{c}$. This is exactly what was observed (Fig. 5(a)).

\subsection{Relation of Observed Behaviour to Dynamic Strain Ageing Effects}

With regard to dynamic strain ageing effects, the enhanced (cyclic) work hardening and the promotion of a planar slip mode in the temperature range of dynamic strain ageing (cf. Introduction), as observed in strained (fatigued) specimens around $400^{\circ} \mathrm{C}^{5,6)}$ are the main features that should be related to variations of the dislocation behaviour with temperature. Among the findings of this study, the weak maximum in the dissociation width of near-screw dislocations introduced by deformation at elevated temperatures at around $400^{\circ} \mathrm{C}$ (Fig. 5(b)) appears to be the only feature that could be associated with the dynamic strain ageing effects mentioned above. Recent work by two of the authors ${ }^{22)}$ on a stainless steel of similar composition as in this work but with a significantly lower carbon content $(<0.01 \%)$ supports the present findings.

The behaviour described above is probably not related primarily to the true dependence of the SFE on temprature but rather to segregation effects, as explained in Sec. 5.2. Nonetheless, the fact remains that, in the temperature range of maximum dynamic strain ageing around $400^{\circ} \mathrm{C}$, the near-screw dislocations are more widely dissociated than at lower or higher temperatures. Thus, one could expect reduced cross slip which in turn would mean that the slip mode should become more planar. Also, dynamic recovery due to mutual trapping and annihilation of unlike near-screw dislocations by cross slip should be reduced, giving rise to enhanced work hardening. Both types of behaviour would be in qualitative agreement with the described observations in the temperature regime of dynamic strain ageing.

However, the temperature dependence of the observed variation in the dissociation width of screw dislocations (Fig. 5(b)) is rather weak, and other effects such as the formation of small coherent shearable carbides and the occurrence of short range ordering can also enhance planar slip, as pointed out in the Introduction. Hence, it is suggested that the observed effects are only partially responsible for the temperature dependence of the slip mode.

\section{REFERENCES}

1) C. F. Jenkins and G. V. Smith: Trans. Metall. Soc. AIME, 245 (1969), 2149.

2) K. Onishi and J. Ishizuka: J. Iron Steel Inst. Jpn., 63(1977), 2362.

3) B. P. Kashyap, K. McTaggart and K. Tangri: Philos. Mag., A, 57 (1988), 97.

4) K. Tsuzaki, T. Hori, T. Maki and I. Tamura: Mater. Sci. Eng., 61 (1983), 247.

5) F. Petry, H.-J. Christ and H. Mughrabi: Gefüge und Mechanische Eigenschaften von Werkstoffen, DGM Informationsgesellschaft, Oberursel, (1991), 79.

6) R. Zauter, F. Petry, H.-J. Christ and H. Mughrabi: Thermomechanical Fatigue Behaviour of Materials, ed. by $\mathrm{H}$. Sehitoglu, ASTM STP 1186, Amer. Soc. Test. Mater., Philadelphia, (1993), 70

7) C. E. Feltner and C. Laird: Trans. Metall. Soc. AIME, 242 (1968), 1253.

8) V. Gerold and H.-P. Karnthaler: Acta Metall., 37 (1989), 2177.

9) C. G. Rhodes and A. W. Thompson: Metall. Trans. A, 8 (1977) 1901.

10) C. C. Bampton, I. P. Jones and M. H. Loretto: Acta Metall., 26 (1978), 39.

11) R. M. Latanision and A. W. Ruff: Metall. Trans., 2 (1971), 505.

12) F. Lecroisey and A. Pineau: Metall. Trans., 3 (1972), 387.

13) F. Abrassart: Metall. Trans., 4 (1973), 2205.

14) D. J. H. Cockayne, M. L. Jenkins and I. L. F. Ray: Philos. Mag., 24 (1971), 1383.

15) A. Head, P. Humble, L. M. Clarebrough, A. J. Morton and C. T. Forwood: Computed Electron Micrographs and Defect Identification, Amsterdam, North-Holland, (1973).

16) V. K. Salmutter and E. Stangler: Z. Metall., 51 (1960), 544.

17) H. Saka, Y. Sueki and T. Imura: Philos. Mag. A, 37 (1978), 273.

18) H. Saka: Philos. Mag. A, 47 (1983), 131.

19) P. C. Gallagher: Metall. Trans., 1 (1970), 2429.

20) H. Saka, T. Kondo and T. Imura: Philos. Mag. A, 47 (1983), 859.

21) L. Remy, A. Pineau and B. Thomas: Mater. Sci. Eng., 36 (1978), 47.

22) Y. Kaneko and H. Saka: to be published. 